\title{
AUTOESTIMA E SATISFAÇÃO SEXUAL APÓS COMPLICAÇÕES CIRÚRGICAS DA PROSTATECTOMIA RADICAL
}

\section{Self-esteem and sexual satisfaction after surgical complications of radical prostatectomy Autoestima y satisfacción sexual tras complicaciones quirúrgicas de la prostatectomía radical} Débora Moura Miranda Goulart', Mário Alfredo Silveira Miranzi', Paulo Eduardo Nunes Goulart ${ }^{3}$

RESUMO: Objetivo: Comparar as medidas de autoestima e de satisfação sexual segundo a presença de disfunção erétil (DE) e incontinência urinária (IU) em pacientes submetidos à prostatectomia radical $(\mathrm{PR})$, nos primeiros cinco anos do pós-operatório. Método: Estudo transversal, com análise descritiva de 81 pacientes submetidos à PR. Resultados: A média etária foi de 65,5 anos. A maioria dos pacientes (85,2\%) procurou a urologia assintomáticos e 76,6\% possuíam tumores localizados de alto risco. Um número considerável referiu hipertensão arterial $(53,1 \%)$. Após a cirurgia, os pacientes apresentaram $\mathrm{DE}(90,1 \%)$ e IU $(33,3 \%)$. A maioria (70,4\%) tinha desempenho de nulo a ruim. Foi observado pior escore de autoestima ( $\mathrm{p}=0,019)$ e pior desempenho sexual $(\mathrm{p}<0,001)$ em pacientes com DE. Conclusão: As possíveis sequelas da PR podem alterar a sexualidade e consequentemente impactar a autoestima. Palavras-chave: Prostatectomia. Disfunção erétil. Incontinência urinária. Autoimagem.

ABSTRACT: Objective: To compare the measurements of self-esteem and sexual satisfaction according to the presence of erectile dysfunction (ED) and urinary incontinence (UI) in patients submitted to radical prostatectomy (RP), in the five first postoperative years. Method: Cross-sectional study with a descriptive analysis of 81 patients submitted to RP. Results: Mean age was 65.5 years. Most patients (85.2\%) sought urology care while being asymptomatic, and $76.6 \%$ had high-risk tumors. A considerable number of patients reported arterial hypertension (53.1\%). After surgery, patients presented with ED (90.1\%) and UI (33.3\%). A majority of patients (70.4\%) had zero to poor performance. A worse self-esteem score was observed (p=0.0190), as well as worse sexual performance $(\mathrm{p}<0.001)$ among patients with ED. Conclusion: The possible after affects of RP may change sexuality and, consequently, have an impact on self-esteem.

Keywords: Prostatectomy. Erectile dysfunction. Urinary incontinence. Self concept.

RESUMEN: Objetivo: Comparar las medidas de autoestima y de satisfacción sexual según la presencia de disfunción eréctil (DE) e incontinencia urinaria (IU) en pacientes sometidos a la prostatectomía radical (PR), en los primeros cinco años del pos-operatorio. Método: Estudio transversal, con análisis descriptiva de 81 pacientes sometidos a la PR. Resultados: El promedio de edad fue de 65,5 años. La mayoría de los pacientes (85,2\%) buscó la urología asintomática y un $76,6 \%$ poseían tumores localizados de alto riesgo. Un número considerable refirió hipertensión arterial (53,1\%). Tras la cirugía, los pacientes presentaron DE $(90,1 \%)$ e IU (33,3\%). La mayoría (70,4\%) tenía desempeño de nulo a malo. Fue observado peor escore de autoestima ( $\mathrm{p}=0,019)$ y peor desempeño sexual $(\mathrm{p}<0,001)$ en pacientes con $\mathrm{DE}$. Conclusión: Las posibles secuelas de la PR pueden alterar la sexualidad y consecuentemente impactar la autoestima.

Palabras clave: Prostatectomía. Disfunción eréctil. Incontinencia urinaria. Autoimagen.

'Enfermeira. Mestre em Atenção à Saúde pela Universidade Federal do Triângulo Mineiro (UFTM). Docente do curso de enfermagem da Universidade UniEvangélica - Anápolis (G0), Brasil. E-mail: debysmm@gmail.com

Rua Manelico Crispim Qd 51 Lt27 - Jundiaí - CEP: 75110-450 - Anápolis (G0), Brasil.

2Odontólogo. Doutor em Saúde Coletiva pela Unicamp. Professor Adjunto da UFTM) - Uberaba (MG), Brasil.

${ }^{3}$ Médico. Urologista pela UFTM - Uberaba (MG), Brasil.

Recebido: 11 out. 2016 - Aprovado: 16 dez. 2016

DOI: $10.5327 / Z 1414-4425201700010005$ 


\section{INTRODUÇÃO}

Atualmente, o câncer é uma das principais causas de morte no mundo, sendo considerado um problema de saúde pública em países desenvolvidos e em desenvolvimento. A Organização Mundial da Saúde estimou que, para o ano de 2030, são esperados 27 milhões de casos incidentes de câncer, 17 milhões de mortes por câncer e 75 milhões de pessoas convivendo, anualmente, com câncer. A última estimativa mundial apontou o câncer da próstata $(\mathrm{CaP})$ como sendo o segundo tipo de câncer mais frequente em homens, com cerca de 1,1 milhão de casos novos no ano de $2012^{1}$.

No Brasil, a estimativa para o ano de 2016, válida também para o ano de 2017, reforça a magnitude do problema. São esperados, aproximadamente, 596 mil casos novos, sendo 61.200 de $\mathrm{CaP}$, que continua sendo a segunda neoplasia mais comum entre os homens. O aumento da expectativa de vida, a evolução dos métodos diagnósticos e a melhora da qualidade dos sistemas de informação do país podem explicar o aumento das taxas de incidência ao longo dos anos ${ }^{2}$. Isso significa que, na verdade, não se trata de um aumento no número de ocorrências da enfermidade, mas uma maior capacidade de se efetuar o diagnóstico da doença e uma maior preocupação da população em relação a sua saúde.

Achados no exame clínico, combinados com o resultado da dosagem sérica do PSA (Prostatic Specific Antigen), podem sugerir a existência da doença. Nos casos suspeitos, é indicada biópsia prostática com relatório anatomopatológico para fornecer a graduação histológica de Gleason. O objetivo é identificar a provável taxa de crescimento tumoral e sua tendência à disseminação, além de ajudar a definir o melhor tratamento para o paciente ${ }^{3}$.

O CaP é uma doença heterogênea com diferentes características de comportamento e, por isso, define subgrupos com alto risco de recorrência após tratamento local. A fim de simplificar a interpretação dos dados antes do tratamento e definir a estratégia mais apropriada para cada paciente, D’Amico propôs a estratificação de grupos de risco em três categorias (baixo, intermediário e alto) que usam o valor do PSA ao diagnóstico, o grau histológico de Gleason e o estadiamento clínico 4 .

Os pacientes com $\mathrm{CaP}$ devem ser tratados em função do estágio da doença e, nesse sentido, a prostatectomia radical (PR) é considerada padrão ouro para o tratamento do $\mathrm{CaP}$ nos casos de tumores restritos à próstata, com excelentes índices de sobrevida ${ }^{4}$.
Todas as modalidades terapêuticas apresentam risco significativo e efeitos colaterais, alterando com impacto negativo a qualidade de vida $(\mathrm{QV})$ dos pacientes. No caso da $\mathrm{PR}$, as principais complicações relacionam-se com estenose uretral, incontinência urinária (IU) e disfunção erétil (DE). Estudos apontam esses dois últimos como os principais fatores comprometedores da QV após a $\mathrm{PR}^{3}$.

Alterações de comportamento podem influenciar de forma decisiva a QV, a evolução da doença e o prognóstico. É fundamental que o diagnóstico seja associado a um exame psicodiagnóstico para adequado tratamento, inclusive quanto à aceitação da doença e a como lidar com os sentimentos que emergem nesse momento ${ }^{3}$.

A PR e a possibilidade de sequelas incrementam e facilitam o aparecimento de medos relacionados à impotência, perdas e morte. A deteç̧ão precoce e o tratamento devem considerar os aspectos emocionais envolvidos; afinal, eles propiciam a emergência de conflitos sexuais e constituem-se uma ameaça à integridade e à identidade masculina ${ }^{3}$.

O sentimento de impotência causa repercussões na vida dos pacientes com CaP. Embora haja evolução em termos de possibilidades de tratamento, por se tratar de um órgão que afeta a sensibilidade sexual masculina, o sentimento de impotência está presente mesmo naqueles com impotência transitória. Alterações emocionais, como depressão, afetam a sexualidade, que modifica a fisiologia da ereção. Uma falha erétil pode antecipar o fracasso e produzir ou aumentar a ansiedade ${ }^{5}$.

Percebe-se que os tumores urogenitais acarretam implicações psicológicas adicionais, principalmente por parte dos homens. Além de importantes consequências emocionais trazidas pelas questões sobre cura e morte, é comum diagnóstico e tratamento envolverem perda da masculinidade, queda da autoestima (AE), confronto com a perspectiva de mudanças na vida, imagem corporal, relações pessoais e sofrimento, que afetam o bem-estar físico e emocional, comprometendo a QV em geral .

Nesse sentido, a população masculina com câncer necessita da atenção. O papel do enfermeiro na promoção da saúde e na reabilitação de pacientes pós-prostatectomias radicais é fundamental, uma vez que lesões nervosas decorrentes desse tipo de procedimento podem determinar disfunções eréteis e incontinência urinária. Cabe ao enfermeiro uma observação meticulosa em busca de estratégias que possibilitem o alcance das necessidades da saúde desse homem, e oferecer subsídios com vistas a uma melhor QV 5 .

Frente aos efeitos do diagnóstico e do tratamento cirúrgico, conhecer o perfil dos pacientes para elaborar um plano 
de assistência torna-se fundamental. Nesse sentido, surge o questionamento: as principais complicações cirúrgicas da PR, a DE e a IU, interferem na autoestima e na satisfação sexual dos pacientes no pós-operatório?

\section{OBJETIVO}

Comparar as medidas de autoestima e de satisfação sexual segundo a presença de DE e IU, nos cinco primeiros anos do pós-operatório, em pacientes com $\mathrm{CaP}$ submetidos à $\mathrm{PR}$.

\section{MÉTODOS}

Trata-se de um estudo observacional, descritivo e correlacional, tipo corte transversal. A população participante da pesquisa foi constituída por 187 pacientes submetidos à $\mathrm{PR}$ no período de setembro de 2006 a setembro de 2011, em um hospital especializado em oncologia.

Participaram do estudo 81 pacientes que atenderam aos seguintes critérios de inclusão: residir em Uberaba (MG); possuir diagnóstico de CaP localizado ou localmente avançado; ter sido submetido à $\mathrm{PR}$ retropúbica com ou sem linfadenectomia; apresentar condições cognitivas para compreender as questões; e ter período mínimo de três meses de cirurgia. Os critérios de exclusão foram: ter diagnóstico psiquiátrico ou fazer uso de medicamentos com efeitos no sistema nervoso central; ter sido submetido à PR de resgate ou ter participado de terapia adjuvante e não possuir informação sobre o estádio clínico do tumor no prontuário.

Antes da coleta de dados, foi realizado teste piloto, sem reposição. Por meio do arquivo médico do hospital, obtevese a relação dos pacientes que estavam incluídos no período escolhido. Mediante aleatoriedade simples, foram sorteados cinco indivíduos. A coleta de dados foi iniciada após conclusão do teste. Os pesquisadores, por meio de contato telefônico, convidaram os pacientes a comparecer ao serviço em dia e local combinados, para a aplicação dos instrumentos. Nessa ocasião, foram explicados os objetivos e a importância da pesquisa, o direito à não participação sem qualquer prejuízo ao tratamento, o anonimato, o sigilo e a privacidade.

A primeira parte da coleta de dados foi realizada em uma sala ambulatorial do hospital, previamente reservada e fora do horário de funcionamento dos ambulatórios de rotina. Tal estratégia visou garantir a privacidade e conseguir, em tempo hábil, o retorno de um maior número de pacientes. Os questionários foram aplicados por um profissional de saúde, masculino, responsável pelo acompanhamento clínico do paciente. As perguntas foram realizadas em encontro único e em situação de privacidade, após a leitura e a assinatura do Termo de Consentimento Livre e Esclarecido (TCLE).

A segunda parte da coleta foi desenvolvida no serviço de arquivo médico do hospital, com consulta ao prontuário a fim de obter informações sobre a história clínica e cirúrgica dos pacientes.

As variáveis sociodemográficas e clínico-cirúrgicas estudadas foram: data de nascimento (dia, mês e ano); presença de hipertensão arterial (HAS), diabetes mellitus (DM) e doenças cardíacas; e data da cirurgia e classificação de risco segundo D’Amico. Para a obtenção dos escores de DE, IU, AE e desempenho sexual, foram utilizados os seguintes instrumentos: International Index of Erectile Function (IIEF-5), International Consultation on Incontinence Questionnaire Short Form (ICIQ-SF), Escala de Autoestima de Rosenberg (EAR) e Quociente Sexual - Versão Masculina (QS-M).

O IIEF-5 é um questionário com 15 itens, traduzido para o português e validado como uma escala autoaplicável para avaliar a função sexual. É válido do ponto de vista psicométrico e de fácil aplicação em pesquisas de ambiente clínico. Cinco fatores são identificados: função erétil, função orgásmica, desejo sexual, satisfação na relação sexual e satisfação global. De acordo com as respostas, a disfunção erétil pode ser classificada de grave a ausente ${ }^{6}$.

O ICIQ-SF, validado e traduzido para o português, apresenta propriedades psicométricas como validade, confiabilidade e responsividade para ambos os sexos. É composto por 6 questões, sendo o escore final ( 0 a 21 ) a soma dos escores das questões 3 , 4 e 5 . Neste estudo, foram descartadas as duas primeiras questões, permanecendo as três questões que compõem o escore e a última, de caráter descritivo. Todos os homens que tiveram soma maior do que três foram considerados incontinentes ${ }^{7}$.

A escala de AE elaborada por Rosenberg (1965) é um instrumento unidimensional que avalia a autoestima pessoal. Trata-se de uma escala do tipo likert de quatro pontos (concordo plenamente, concordo, discordo, discordo plenamente), traduzida para a língua portuguesa, adaptada ao contexto cultural brasileiro e validada. O escore varia de 0 a 30, sendo a baixa AE indicada por altos valores ${ }^{8}$.

Quociente Sexual - Versão Masculina (QS-M) é um questionário elaborado e validado por $\mathrm{Abdo}^{9}$, e contempla componentes físicos e emocionais da função sexual. Seus 
resultados sugerem que o escore total obtido pode distinguir claramente indivíduos com e sem disfunção sexual. As opções de resposta variam de nunca (zero) a sempre (cinco), e o desempenho pode ir de nulo a excelente ${ }^{9}$.

Foi construída uma planilha eletrônica para armazenamento dos dados, por meio do programa Microsoft ${ }^{\circledR}$ Office Excel $^{\circledR} 2007$ (Microsoft Corporation, Redmond, Washington, Estados Unidos). Os dados coletados foram digitados em dupla entrada para posterior verificação da consistência e consolidação. Os dados armazenados e validados no programa Microsoft ${ }^{\circledR}$ Office Excel ${ }^{\circledR} 2007$ foram importados para o aplicativo Statistical Package for Social Sciences (SPSS) versão 16.0.

$\mathrm{Na}$ caracterização da população foram utilizadas medidas descritivas, ou seja, foi realizada a distribuição de frequências para as variáveis categóricas e medidas de centralidade (média e mediana) e de dispersão (desvio padrão, valores mínimo e máximo) para as variáveis numéricas.

Foi realizada categorização da idade em faixas etárias $(<40$; 40 - 50; 50 - 60; 60 - 70; $70-80 ; \geq 80$ ), tornando-as categóricas ordinais. O tempo pós-operatório (variável quantitativa), obtido a partir da data de cirurgia, foi dicotomizado (até um ano de cirurgia e mais de um ano). Houve recategorização dos escores de DE e IU, tornando-as dicotômicas (presença e ausência), e da idade em dois grupos etários (adulto e idoso).

Para comparar as medidas de AE e satisfação sexual, segundo a presença ou ausência de DE e IU, foi realizado o teste não paramétrico Mann-Whitney de acordo com os resultados do teste de normalidade de Shapiro Wilk. O nível de significância adotado foi $\alpha=0,05$. A consistência interna dos instrumentos (IIEF-5 e ICIQ) foi verificada por meio do Coeficiente de alfa de Cronbach.

O projeto foi encaminhado ao Comitê de Ética em Pesquisa com Seres Humanos da UFTM e, após sua aprovação pelo parecer consubstanciado sob protocolo número 2.099/2011, iniciou-se o teste piloto e a coleta dos dados.

\section{RESULTADOS}

Com relação às características sociodemográficas, a idade variou de 37 a 81 anos, sendo a média de 65,5 anos ( $\mathrm{DP}=8,4)$, $\operatorname{com} 77,8 \%$ acima de 60 anos. Em relação às comorbidades, um total de $53,1 \%$ referiram hipertensão arterial; $17,3 \%$, diabetes mellitus; e $27,2 \%$, algum tipo de cardiopatia.

Os pacientes foram questionados sobre os motivos que os levaram a procurar o serviço de urologia. A maioria procurou avaliação prostática de rotina $(85,2 \%)$, enquanto apenas
14,8\% apresentavam sintomas urinários. O tempo médio de pós-operatório foi de 25,9 meses com variação de 6 a 48,4 meses $( \pm 1,3)$, sendo que a maioria tinha realizado a PR há mais de 1 ano (76,5\%). De acordo com a classificação de D'Amico, 76,6\% dos tumores localizados eram de alto risco.

Em relação à DE, o IIEF-5 apresentou média de $6,9( \pm 10,2)$ nas respostas, com variação de escore de 1 a 30 pontos. $O$ alfa de Cronbach de 0,99 indicou elevada consistência interna e homogeneidade dos itens. Foi observado que, após a cirurgia, a maioria dos pacientes não teve atividade sexual $(74,1 \%)$, não tentou penetração $(75,3 \%)$ e, ainda, apresentou muito baixa confiança em relação à ereção $(69,1 \%)$. De acordo com o escore do IIEF5 , a maioria $(90,1 \%)$ dos pacientes apresentou algum grau de $\mathrm{DE}$, sendo DE grave em $75,3 \%$ deles. A associação entre tempo pós-operatório e $\mathrm{DE}$ foi significativamente maior no grupo de idosos ( $\mathrm{p}=0,01$ ). Do total de pacientes com DE, $76,7 \%$ tinham tumores classificados como de alto risco.

Em relação à incontinência urinária, o ICIQ-SF apresentou um alfa de Cronbach de 0,90 ; o que indica elevada consistência interna e homogeneidade dos itens. Observou-se frequência de 33,3\% de IU, com média de escore de 3,26 $( \pm 5,37)$ e variação de 0 a 21 . A maioria tinha feito cirurgia há mais de 1 ano $(70,4 \%)$ e eram idosos (81,5\%). Neste estudo não houve diferenças significativas da IU entre os pacientes com até um ano de cirurgia em relação àqueles com mais de um ano, bem como entre adultos e idosos.

O instrumento de EAR apresentou um alfa de Cronbach de 0,95. A média dos escores das respostas foi de 5,38 ( $\mathrm{DP}=6,6)$ com variação de 0 a 28 pontos, indicando elevada $A E$.

Os dados da Tabela 1 mostram que os pacientes com DE e IU tiveram os maiores postos médios (pior autoestima), sendo essa correlação significativamente maior entre os pacientes com DE $(\mathrm{p}=0,019)$.

Tabela 1. Comparação dos escores de autoestima entre os pacientes submetidos à prostatectomia radical, segundo a presença de disfunção erétil e incontinência urinária. Uberaba (MG), 2012.

\begin{tabular}{|l|c|c|c|}
\hline & Mediana & Posto médio & Valor p $^{*}$ \\
\hline \multicolumn{1}{|c|}{ Disfunção erétil } & \multicolumn{3}{|l}{} \\
\hline Sim & 4,0 & 42,99 & 0,019 \\
\hline Não & 0 & 22,88 & \\
\hline Incontinência urinária & & \\
\hline Sim & 5,0 & 44,37 & 0,350 \\
\hline Não & 1,5 & 39,31 & \\
\hline
\end{tabular}

*Valor p obtido pelo teste não paramétrico de Mann-Whitney. 
O QS-M apresentou um alfa de Cronbach de 0,98. A média dos escores das respostas foi de $20,57(\mathrm{DP}=32,5)$ com variação de 0 a 100 pontos. A maioria dos pacientes apresentou um padrão de desempenho sexual de nulo a ruim $(70,4 \%)$. Esse resultado pode estar relacionado com as complicações cirúrgicas da PR (Tabela 2).

Os dados mostram que os pacientes sem $\mathrm{DE}$ tiveram maiores postos médios, ou seja, maior satisfação sexual em relação àqueles com $\mathrm{DE}(\mathrm{p}<0,001)$. Os pacientes sem IU também apresentaram postos médios maiores, indicando que possuem um melhor padrão de desempenho sexual, embora não houvesse diferença significativa entre os que tinham ou não IU.

\section{DISCUSSÃO}

O CaP é considerado um câncer da terceira idade, uma vez que três quartos dos casos no mundo ocorrem a partir dos 65 anos. A incidência do $\mathrm{CaP}$ aumenta com a idade, atingindo quase $50 \%$ dos indivíduos com 80 anos, o que indica que esse tumor, provavelmente, não poupará nenhum homem que viver até $100 \operatorname{anos}^{10}$. Segundo o Instituto Nacional de Câncer (INCA), o único fator de risco bem estabelecido para o desenvolvimento do CaP é a idade: aproximadamente $62 \%$ dos casos diagnosticados no mundo acometem homens com 65 anos ou mais. No presente estudo, a média etária foi de 65,5 anos. Resultados semelhantes foram encontrados em outros estudos. Em uma coorte hospitalar composta por pacientes com CaP localizado, a média foi de 73 anos (40 - 87), sendo 63 anos a média para aqueles que foram submetidos à $\mathrm{PR}^{11}$. Outro estudo mostrou um valor de 66 anos $(43-77)$ com $81 \%$ acima de 60 anos $^{3}$.

Tabela 2. Comparação dos escores do Quociente Sexual - Versão Masculina (QS-M) entre os pacientes submetidos à prostatectomia radical segundo a presença de disfunção erétil e incontinência urinária. Uberaba (MG), 2012.

\begin{tabular}{|c|c|c|c|}
\hline & Mediana & Posto médio & Valor p* \\
\hline \multicolumn{4}{|c|}{ Disfunção erétil } \\
\hline $\operatorname{Sim}$ & 1,0 & 37,21 & \multirow{2}{*}{$<0,001$} \\
\hline Não & 4,5 & 75,63 & \\
\hline \multicolumn{4}{|c|}{ Incontinência urinária } \\
\hline Sim & 1,0 & 38,65 & \multirow{2}{*}{0,458} \\
\hline Não & 1,0 & 42,18 & \\
\hline
\end{tabular}

*Valor p obtido pelo teste não paramétrico de Mann-Whitney.
Existe relação direta e linear da pressão arterial com a idade, sendo a prevalência de HAS superior a $60 \%$ na população acima de 65 anos. Assim, o elevado índice de hipertensos neste estudo pode estar relacionado com a frequência de idosos.

Em relação à etiologia do $\mathrm{CaP}$, são ressaltados os fatores de risco relacionados com a idade e a hereditariedade. Contudo, alguns fatores exógenos podem ter importante papel no desenvolvimento do $\mathrm{CaP}$ e impacto no risco de progressão do câncer latente para a sua forma clínica. Esses fatores geralmente se associam com condições clínicas, tais como HAS, DM e cardiopatias, que podem funcionar como potenciais complicadores no pós-operatório ${ }^{12}$. Assim, os resultados das variáveis clínicas deste estudo são dados importantes que possivelmente se associam com a etiologia ou com as complicações cirúrgicas (DE e IU).

A maioria dos pacientes no grupo de estudo procurou avaliação prostática de rotina sem qualquer sintomatologia urinária. Uma das peculiaridades do CaP é justamente a capacidade de ser encontrada em um número elevado de indivíduos sem causar qualquer mal. Uma recente revisão sistemática de estudos que avaliaram a próstata por meio de necropsias de homens que faleceram sem doença prostática aparente revelou focos neoplásicos em indivíduos que não apresentaram em vida manifestações clínicas relacionadas ao CaP. O estudo mostra que os tumores no grupo etário de idosos têm caráter indolente, são assintomáticos e seus portadores morrem por outros motivos; ou seja, morrem com o câncer, mas não pelo câncer ${ }^{13}$.

Em relação à DE, foi observado que, após a cirurgia, a maioria dos pacientes não teve atividade sexual, não tentou penetração e apresentou muito baixa confiança em relação à ereção, sendo que a maioria mostrou algum grau de DE. Esses dados coincidem com os da literatura, na qual as taxas de impotência relatadas são discrepantes e altas, variando entre 60 e $90 \%{ }^{14}$.

A idade do paciente no momento da cirurgia é essencial para determinar a recuperação da função erétil após a cirurgia. Estudos têm mostrado a associação entre idade e DE, indicando que melhores taxas de potência no pós-operatório são obtidas na população mais jovem, que também é a mais propensa a ter uma melhor função erétil pré-operatória e estar mais interessada na recuperação sexual após a cirurgia ${ }^{15-17}$. No presente estudo, a DE associou-se ao grupo dos idosos.

A preservação dos feixes nervosos deve ser considerada para pacientes com CaP localizado, sendo que os melhores candidatos são aqueles com tumores de baixo risco segundo D'Amico ${ }^{15}$. Embora não tenha sido avaliada, neste estudo, a função erétil pré-operatória e a preservação dos feixes nervosos, pode-se 
supor que, na maioria dos pacientes, não tenha sido possível tal preservação, uma vez que prevaleceu o grupo de alto risco.

A taxa de IU mostrou-se coerente com a literatura, haja vista as variáveis que geram grande amplitude em seus índices. Vale ressaltar que não foi realizada a classificação da IU e foram considerados incontinentes os pacientes que apresentaram qualquer perda. Taxas de IU variam consideravelmente e isso pode estar relacionado com as diferenças nas abordagens cirúrgicas, nas definições de IU, nas metodologias dos estudos, bem como o tempo de seguimento e instrumentos de avaliação da função urinária. Existe, na literatura, variação de 8 a $77 \%{ }^{18}$. A IU moderada ou grave está presente em 3 a $5 \%$ dos casos quando a intervenção é realizada por equipes habilitadas ${ }^{10}$.

Em relação à $\mathrm{AE}$, houve uma correlação significativamente maior entre os pacientes com DE. Estudos indicam que o envolvimento do sistema urinário e reprodutor e as chances de alterações na continência urinária e na função sexual podem provocar respostas emocionais importantes nos pacientes prostatectomizados ${ }^{5,14}$.

Um estudo prospectivo mostrou que, embora apresentem DE ou IU, os pacientes relatam que não têm problemas ou que têm um pequeno problema. Portanto, apesar de não terem ereções e de perderem urina involuntariamente, esses pacientes aparentemente se adaptaram aos agentes estressores, mantendo uma autoimagem positiva ${ }^{19}$. Quando são associados métodos qualitativos aos estudos, os homens relatam que a DE é propulsora de grande aflição no pós-operatório com expressiva diminuição da $\mathrm{AE}$, como foi verificado em um estudo que observou que homens gostam de mostrar a si mesmos e aos outros que lidam facilmente com problemas, tendendo a diminuir ou negar a existência dos mesmos para evitar preocupar familiares e não serem percebidos pelos outros como vulneráveis ${ }^{20}$.

Os dados sobre a satisfação sexual corroboram com a literatura, que evidencia a relação entre sexualidade, masculinidade e próstata. Um estudo mostrou que homens que apresentaram atividade sexual satisfatória antes da PR e após a cirurgia passaram a apresentar as seguintes características: presença de DE, inibição do desejo, conflito conjugal e masturbação ${ }^{3}$.

Enquanto o funcionamento sexual certamente diminui com a idade, a sexualidade e o sentido de si mesmos como seres sexuais continua a ser um aspecto importante da vida dos homens. Embora o CaP isoladamente possa precipitar uma reavaliação da vida, a DE pode desafiar ainda mais sua identidade e provocar mudanças na forma como os homens veem-se como seres sexuais, como inserem-se na sociedade e na sua relação com as mulheres ${ }^{14}$.
Terapias psicológicas especificamente orientadas para pacientes tratados de CaP são importantes para auxiliar os homens a reconhecer, expressar e aceitar as mudanças causadas pelos tratamentos, para melhorar a comunicação com suas parceiras e com a equipe assistencial, e para orientá-los na busca por soluções adaptativas para problemas urinários e sexuais.

Os cuidados ao paciente com CaP geralmente são decorrentes de um empenho multidisciplinar. A enfermagem exerce um papel fundamental, pois é o grupo profissional que mantém contato com o paciente e sua família em todos os períodos da doença e em diferentes contextos de atendimento. Porém, para fornecer um cuidado com qualidade, é preciso conhecer as dimensões biológicas, fisiológicas e psicológicas da doença, dos tratamentos e do impacto desses na vida dos pacientes e familiares.

É importante elucidar que, embora o objetivo principal de qualquer tratamento para $\mathrm{CaP}$ seja maximizar a esperança de vida, os pacientes e a equipe de saúde precisam estar atentos ao impacto das terapias, já que a sobrevida que não é acompanhada de QV pode não ser a melhor opção para o paciente, causando mais sofrimento.

\section{CONCLUSÃO}

Os resultados obtidos neste estudo permitiram estabelecer as conclusões expostas a seguir:

Quanto à caracterização sociodemográfica:

- A idade variou de 37 a 81 anos, com média de 65,5 $(\mathrm{DP}=8,4)$ e com $77,8 \%$ com 60 anos ou mais.

Quanto à caracterização clínico-cirúrgica:

- Referiram HAS $(53,1 \%)$, DM (17,8\%) e cardiopatias $(27,2 \%)$. A maioria procurou o serviço de urologia enquanto assintomática $(85,2 \%)$. De acordo com a classificação de D’Amico, 76,6\% dos tumores localizados eram de alto risco. Quanto ao tempo pós-operatório, 76,5\% tinha mais de 1 ano de cirurgia.

- Em relação à DE, a média do escore do IIEF-5 $(\alpha=0,99)$ foi de 6,9 $(\mathrm{DP}=10,2)$. A maioria não tinha atividade sexual $(74,1)$, não tentou penetração no pós-operatório $(75,3 \%)$ e tinha baixa confiança em relação à ereção $(69,1 \%)$. A DE estava presente em $90,1 \%$, sendo grave em 75,3\%. Do total de pacientes com DE, $76,7 \%$ tinham tumores classificados como de alto risco;

- O ICIQ-SF $(\alpha=0,90)$ apresentou média de escore de $3,26(\mathrm{DP}=5,37)$ e mostrou IU em $33,3 \%$ dos pacientes. 
- A AE foi considerada elevada considerando os escores da $\operatorname{EAR}(\alpha=0,95)$ que mostrou média de 5,38 (DP=6,6);

- A maioria dos pacientes apresentou padrão de desempenho sexual de nulo a ruim $(70,4 \%)$, considerando o QS-M $(\alpha=0,98)$. A média dos escores foi de 20,57 $(\mathrm{DP}=32,5)$.

Quanto à associação da DE e IU com o grupo etário e tempo pós-operatório:

- Foi observado que, do total de pacientes com DE (73), $82,2 \%$ eram idosos $(p=0,01)$ e $78,1 \%$ tinham mais de
1 ano de cirurgia. Dentre aqueles com IU (27), 81,5\% eram idosos e 70,4\% havia submetido-se à cirurgia há mais de 1 ano.

A comparação das medidas de AE, de satisfação sexual segundo a presença de DE e IU:

- Em relação à $\mathrm{AE}$, apresentaram maiores postos médios (pior AE) os pacientes com DE $(\mathrm{p}=0,019)$ e pacientes com IU;

- Melhor desempenho sexual foi verificado nos pacientes sem DE $(p<0,001)$ e sem IU.

\section{REFERÊNCIAS}

1. Instituto Nacional de Câncer [Internet]. Rio de Janeiro. 2016 [acesso em 29 set 16]. Estimativa 2016: incidência de câncer no Brasil. Disponível em: http://www.inca.gov.br/estimativa/2016/index.asp?ID=2

2. Instituto Nacional de Câncer [Internet]. Rio de Janeiro. 2016 [acesso em 29 set 16]. Tipos de câncer: próstata. Disponível em: http://www2.inca. gov.br/wps/wcm/connect/tiposdecancer/site/home/prostata+/definicao

3. Vieira ACOA. O impacto da doença e tratamento cirúrgico em homens acometidos por câncer de próstata: estudo exploratório de qualidade de vida. [Dissertação]. [São Paulo]: Faculdade de Medicina/USP, 2010.113p.

4. Sociedade Brasileira de Cirurgia Oncológica (SOBOC). Manual de condutas 2011. Câncer de próstata clinicamente localizado. [Internet]. 2011. [acesso em 15 dez 16]. Disponível em: http://www.sboc.org. br/downloads/manual_condutas_2011.pdf

5. Oliveira RDP, Santos MCL, Rocha SR, Braga, VAB, Alves e Souza AM. Aspectos emocionais póstratamento do câncer de próstata: uma revisão integrativa da literatura. Braz J Nurs. 2014;13(4):1-9.

6. Pechorro OS, Calvinho AM, Pereira MN, Vieira RX. Validação de uma versão portuguesa do Índice Internacional de Função Eréctil-5 (IIEF5). Rev Int Androl. 2011;9(1):3-9.

7. Tamanini JTN, Dambros M, D'Ancona CAL, Palma PCR, e Netto Junior NR. Validação para o português do "International Consultation on Incontinence Questionnaire - Short Form" (ICIQ-SF). Rev Saúde Pública. 2004;38(3):438-44.

8. Dini GM, Quaresma MR, Ferreira LM. Adaptação cultural e validação da versão brasileira da escala de autoestima de Rosenberg. Rev Bras Cir Plást. 2004;19(1):41-52.

9. Abdo CHN. Elaboração e validação do quociente sexual versão masculina, uma escala para avaliar a função sexual do homem. Rev Bras Med. 2006;63(2):42-6.

10. Srougi M, Ribeiro LA, Piovesan AC, Colombo JR, Nesrallah A. Doenças da próstata. Rev Med. 2008;87(3):166-77.
11. Araújo JA, Conceição VM, Oliveira RAA, Zago MMF. Caracterização social e clínica dos homens com câncer de próstata atendidos em um hospital universitário. Rev Min Enferm. 2015;19(2):196-203.

12. Medeiros AP, Menezes MFB, Napoleão AA. Fatores de risco e medidas de prevenção do câncer de próstata: subsídios para a enfermagem. Rev Bras Enferm. 2011;64(2):385-8.

13. Bell KJ, Del Mar C, Wright G, Dickinson J, Glasziou P. Prevalence of incidental prostate cancer: a systematic review of autopsy studies. Int J Cancer. 2015;137(7):1749-57.

14. Thompson I, Thrasher JB, Aus G, Burnett AL, Canby-Hagino ED, Cookson MS, et al. Guidelines for the management of clinically localized prostate cancer: 2007 update. J Urol. 2007;177(6):2106-31.

15. Briganti A, Capitanio U, Chun FK, Karakiewicz PI, Salonia A, Bianchi $\mathrm{M}$, et al. Prediction of sexual function after radical prostatectomy. Cancer, New York, 2009;1 15(13):3150-9.

16. Johansson E, Steineck G, Holmberg L, Johansson JE, Nyberg T, Ruutu $M$, et al. Long-term quality-of-life outcomes after radical prostatectomy or watchful waiting: the Scandinavian Prostate Cancer Group-4 randomised trial. The Lancet Oncology 2011;12(9):891-9.

17. Sivarajan G, Prabhu V, Taksler GB, Laze J, Lepor H. Ten-year outcomes of sexual function after radical prostatectomy: results of a prospective longitudinal study. Eur Urol. 2014 Jan;65(1):58-65.

18. Klingler C, Marberger M. Incontinence after prostatectomy: surgical treatment options. Curr Opin Urol. 2006;16(2):60-4.

19. Hoffman RM, Hunt WC, Gilliland FD, Stephenson RA, Potosky AL. Patient satisfaction with treatment decisions for clinically localized prostate carcinoma. Results from the Prostate Cancer Outcomes Study. Cancer, New York, 2003;97(7):1653-62.

20. Eilat-Tsanani S, Tabenkin H, Shental J, Elmalah I, Steinmetz D. Patients' perceptions of radical prostatectomy for localized prostate cancer: a qualitative study. Isr Med Assoc J. 2013;15(3):153-7. 